\title{
Convolution Neural Networks for Localization of Near-Field Sources via Symmetric Double-Nested Array
}

\author{
Xiaolong Su $(\mathbb{D}$, Panhe Hu $(\mathbb{D}$, Zhenghui Gong $(\mathbb{D}$, Zhen Liu $(\mathbb{D}$, Junpeng Shi $\mathbb{D}$, and Xiang Li $\mathbb{D}$ \\ College of Electronic Science and Technology, National University of Defense Technology, Changsha 410073, China \\ Correspondence should be addressed to Panhe Hu; hupanhe13@nudt.edu.cn
}

Received 21 March 2021; Accepted 2 June 2021; Published 10 June 2021

Academic Editor: Jian Feng Li

Copyright () 2021 Xiaolong Su et al. This is an open access article distributed under the Creative Commons Attribution License, which permits unrestricted use, distribution, and reproduction in any medium, provided the original work is properly cited.

\begin{abstract}
We present the convolution neural networks (CNNs) to achieve the localization of near-field sources via the symmetric doublenested array (SDNA). Considering that the incoherent near-field sources can be separated in the frequency spectrum, we first calculate the phase difference matrices and consider the typical elements as the inputs of the networks. In order to guarantee the precision of the angle-of-arrival (AOA) estimation, we implement the autoencoders to divide the AOA subregions and construct the corresponding classification CNNs to obtain the AOAs of near-field sources. Then, we construct a particular range vector without the estimated AOAs and utilize the regression CNN to obtain the range parameters of near-field sources. The proposed algorithm is robust to the off-grid parameters and suitable for the scenarios with the different number of near-field sources. Moreover, the proposed method outperforms the existing method for near-field source localization.
\end{abstract}

\section{Introduction}

Neural networks are developing rapidly with deep learning, which have important applications in the field of array signal processing. By treating the assignment of angle-of-arrival (AOA) label as a separate binary classification, a convolutional neural network $(\mathrm{CNN})$ method in [1] is presented for multispeaker AOA estimation. By employing an end-to-end regression rather than a classification, the complex-valued residual network (ResNet) in [2] is proposed to improve the training performance for AOA estimation in shortrange multiple-input multiple-output (MIMO) communications. In [3], some small deep feedforward networks are trained for AOA estimation under uniform circular array, which can reduce computational complexity and have similar satisfactory performance. In the case of the rectilinear or strictly noncircular sources, a symmetric uniform linear array of cocentered orthogonal loop and dipole (COLD) antennas is exploited in [4] to achieve the localization of near-field sources, where the DOA, range, and polarization parameters are separated by employing the rank reduction (RARE) principle. Moreover, by using the noncircular information for a symmetric uniform linear array, a novel method is proposed in [5] to realize the localization and classification of the mixed sources. Considering that the model-driven approaches depend on the preformulated models $[6,7]$, the data-driven approach of deep neural network (DNN) [8] implements autoencoders and classifiers to estimate the AOA, which can adapt to the scenarios of array imperfections. However, in order to satisfy the additive property when multiple sources are distributed in different subregions, this algorithm replaces the activation function with unit operation, which makes it impossible to approximate the nonlinear model perfectly. Furthermore, the deep convolution network (DCN) [9] is utilized to learn the features of the spectrum proxy and reconstruct the spatial spectrum, which is more accurate and efficient for AOA estimation.

Recent researches have focused on the near-field source localization [10-12], and the regression approach of the CNN structure [13] is proposed to estimate the AOA and range parameters of near-field sources, which has better performance in the case of low signal-to-noise ratios (SNRs) and small number of snapshots. However, this method regards the elements of the covariance matrix as the input of the network, which is only suitable for the scenarios with the fixed number of near-field sources. In contrast, the incoherent 
sources can be separated in the frequency spectrum, employing the phase matrix as the input of the network can be suitable for the different number of sources.

In the case of the same number of sensors, the nested array [14-16] has a larger aperture than the uniform array, which can improve the accuracy of parameter estimation. In [17], the sensor's location of the symmetric doublenested array (SDNA) is given in a closed form. The vectorization of multiple fourth-order cumulant matrices in [18] is applied to achieve the localization of underdetermined near-field sources via a nested array, which can enhance degrees-of-freedom. However, the process of the fourthorder cumulant matrices requires high computational cost.

Accordingly, the motivation of the work is to employ the CNN to achieve near-field source localization and improve the accuracy of parameter estimation. We first calculate the phase difference matrix via the symmetric double-nested array (SDNA). Then, the autoencoders are used to divide the AOA subregions. Next, the corresponding classification CNNs of subregions are constructed to obtain the AOAs of near-field sources. Finally, we employ the regression CNN to determine the range of near-field sources. Simulation results illustrate that the proposed method is robust to the off-grid parameters and suitable for the scenarios with the different number of sources. Moreover, the proposed method outperforms the existing method for near-field source localization.

\section{Signal Model}

As shown in Figure 1, the geometry of SDNA is composed of two subarrays, where the solid circles denote the first subarray with spacing $d$ and locate in

$$
\left\{\xi_{m} \mid \xi_{m}=m d, m=\frac{-M}{2}, \cdots,-2,-1,0,1,2, \cdots, \frac{M}{2}\right\} .
$$

$M$ is even, and the hollow circles denote the second subarray with spacing $(M / 2+1) d$ and locate in

$$
\left\{\xi_{m} \mid \xi_{m}=m\left(\frac{M}{2}+1\right) d, m=\frac{-M}{2}, \cdots,-2,-1,1,2, \cdots, \frac{M}{2}\right\} .
$$

Assuming that the center of the array is regarded as the reference point and the SDNA is impinged by $K$ incoherent narrowband near-field sources, the location of the $k$ th nearfield source can be described as $\left(\theta_{k}, r_{k}\right)$, where $\theta_{k}$ denotes the AOA and $r_{k}$ denotes the range parameter. Note that the near-field region of the array is between $0.62\left(D^{3} / \lambda\right)^{1 / 2}$ and $2 D^{2} / \lambda$, where $D=M(M / 2+1) d$ denotes the aperture of the array and $\lambda$ denotes the wavelength of the near-field sources.

Hence, the received data of the $m$ th sensor at the $n$th snapshot can be modeled as

$$
x_{m}(n)=\sum_{k=1}^{K} s_{k}(n) e^{j \varphi_{k, m}}+w_{m}(n)
$$

for $m=-M, \cdots,-1,0,1, \cdots, M, \quad n=1,2, \cdots, N$, where $N$ denotes the number of snapshots, $s_{k}(n)$ denotes the $k$ th transmitted near-field source at the $n$th snapshot, $w_{m}(n)$ stands for the additive white complex Gaussian noise, and $\varphi_{k, m}$ stands for the phase which is related to the time delay between the $k$ th near-field source at the $m$ th sensor and that at the center of SDNA. In addition, $\varphi_{k, m}$ can be expressed as

$$
\varphi_{k, m}=\frac{2 \pi}{\lambda}\left(r_{k, m}-r_{k}\right)=\frac{2 \pi}{\lambda}\left(\sqrt{r_{k}^{2}+\xi_{m}^{2}-2 r_{k} \xi_{m} \sin \theta_{k}}-r_{k}\right)
$$

for $k=1, \cdots, K$, where $2 \pi / \lambda$ denotes the wavenumber, $r_{k, m}$ denotes the range measured from the $k$ th near-field source to the $m$ th sensor. Applying the second-order Taylor series expansion $[17,18]$ of $r_{k, m}$ at the point $d / r_{k}$, the signal model can be written as

$$
x_{m}(n)=\sum_{k=1}^{K} s_{k}(n) e^{j \xi_{m} \eta_{k}+j \xi_{m}^{2} \phi_{k}}
$$

where $\eta_{k}=-2 \pi \sin \theta_{k} / \lambda$ with $\phi_{k}=\pi \cos ^{2} \theta_{k} / \lambda r_{k}$.

\section{Proposed Algorithm}

In this section, we consider the phase difference as the input of the networks, utilize the autoencoder to divide the AOA subregions, and exploit the CNN to achieve the localization of the near-field sources.

3.1. Preprocessing of Phase Difference. It is generally known that the number of incoherent sources can be estimated by the number of peaks in the frequency spectrum. Assuming that the $g$ th training data contains a single near-field source, the phase difference between the $m_{1}$ th sensor and $m_{2}$ th sensor can be calculated as

$u_{m_{1}, m_{2}, g}= \begin{cases}\vartheta_{m_{1}, g}-\vartheta_{m_{2}, g}+2 \pi & \left(\vartheta_{m_{1}, g}-\vartheta_{m_{2}, g}<-\pi\right), \\ \vartheta_{m_{1}, g}-\vartheta_{m_{2}, g} & \left(-\pi \leq \vartheta_{m_{1}, g}-\vartheta_{m_{2}, g} \leq \pi\right), \\ \vartheta_{m_{1}, g}-\vartheta_{m_{2}, g}-2 \pi & \left(\vartheta_{m_{1}, g}-\vartheta_{m_{2}, g}>\pi\right),\end{cases}$

for $\quad m_{1}, m_{2}=-M, \cdots,-2,-1,0,1,2, \cdots, M, \quad g=1,2, \cdots, G$, where $G$ denotes the number of the training dataset, and $\vartheta_{m_{1}, g}$ and $\vartheta_{m_{2}, g}$, respectively, denote the phase of the peak in frequency spectrum at the $m_{1}$ th sensor and the $m_{2}$ th sensor.

Accordingly, the phase difference is converted between $-2 \pi$ and $2 \pi$, which can be expressed as

$$
u_{m_{1}, m_{2}, g}=\left(\xi_{m_{1}}-\xi_{m_{2}}\right) \eta_{k}+\left(\xi_{m_{1}}^{2}-\xi_{m_{2}}^{2}\right) \phi_{k}
$$

Therefore, the $(2 M+1) \times(2 M+1)$ dimensional phase 


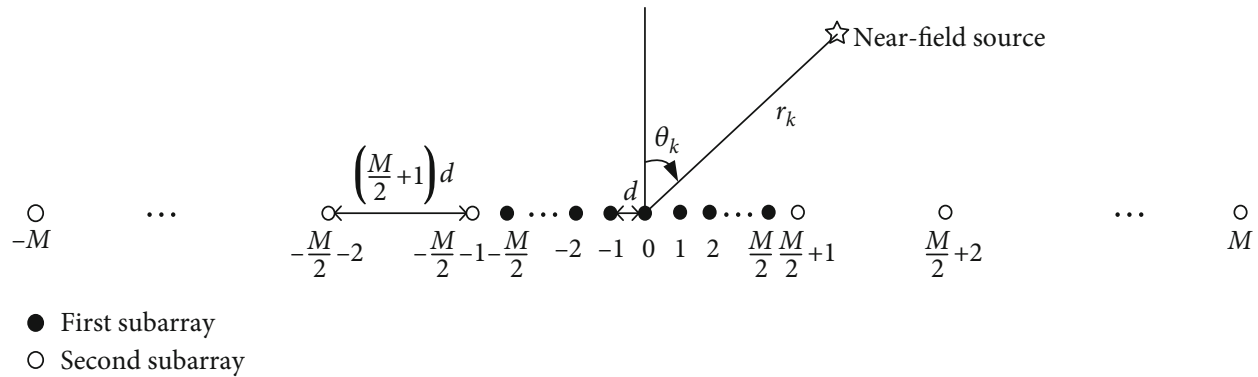

FIgURE 1: Geometry of SDNA with near-field source.

difference matrix of the $g$ th training data can be written as

$$
U_{g}=\left[\begin{array}{ccccc}
u_{-M,-M, g} & \cdots & u_{-M, 0, g} & \cdots & u_{-M, M, g} \\
\vdots & \ddots & \vdots & \ddots & \vdots \\
u_{0,-M, g} & \cdots & u_{0,0, g} & \cdots & u_{0, M, g} \\
\vdots & \ddots & \vdots & \ddots & \vdots \\
u_{M,-M, g} & \cdots & u_{M, 0, g} & \cdots & u_{M, M, g}
\end{array}\right]
$$

where the element $u_{m_{1}, m_{2}, g}$ is calculated by (6). Note that the localization parameters can be determined from the corresponding phase difference matrix.

3.2. Autoencoder of AOA Subregions. Since the counterdiagonal elements of the phase difference matrix in (8) are centrosymmetric and only contain the AOAs but not the range parameters of near-field sources, we first employ the upper right counter-diagonal elements to estimate the AOA of each near-field source.

In order to guarantee the precision of the AOA estimation, we divide the AOA scope into $P$ subregions by utilizing the structure of the autoencoder. The autoencoder of the $p$ th subregion for the $g$ th training data is shown in Figure 2, where the input of the encoder $u_{g}^{(1,0)}$ is an $M$-dimensional vector, the output of the encoder $u_{g}^{(1, p, 1)}$ is an $M / 2$-dimensional vector, and the output of the decoder $u_{g}^{(1, p, 2)}$ is an $M$ -dimensional vector.

Based on the phase difference matrix in (8), the input of the autoencoder is given by

$$
u_{g}^{(1,0)}=\left[u_{-M, M, g}, u_{-M+1, M-1, g}, \cdots, u_{-m_{3}, m_{3}, g}, \cdots, u_{-1,1, g}\right]^{T},
$$

for $m_{3}=M, M-1, \cdots, 1,(\cdot)^{T}$ denotes the transpose operation. To be specific, the output of the encoder is

$$
u_{g}^{(1, p, 1)}=\operatorname{Re} \operatorname{LU}\left(Q_{g}^{(1, p, 1)} u_{g}^{(1,0)}+b_{g}^{(1, p, 1)}\right)
$$

and the output of the decoder is

$$
u_{g}^{(1, p, 2)}=\operatorname{Re} \operatorname{LU}\left(Q_{g}^{(1, p, 2)} u_{g}^{(1, p, 1)}+b_{g}^{(1, p, 2)}\right)
$$

where $\operatorname{ReLU}(\cdot)$ denotes the activation function of a rectified

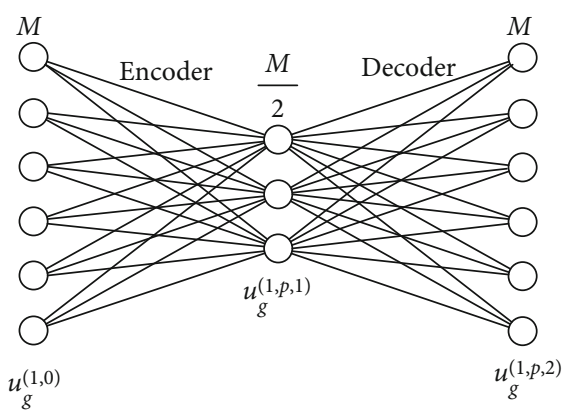

Figure 2: Autoencoder of the $p$ th subregion for the $g$ th data.

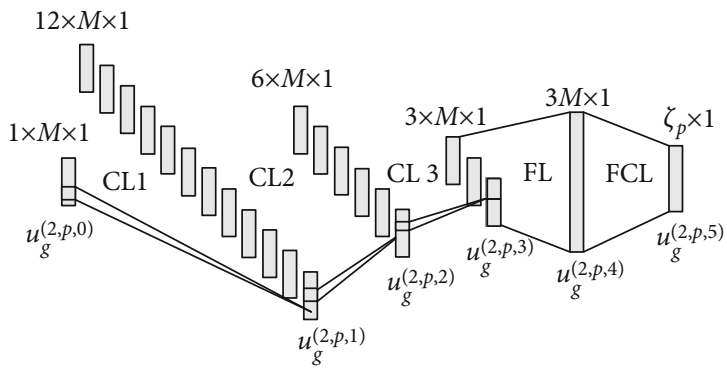

FIgURe 3: Classification CNN for the $p$ th subregion of the $g$ th data.

linear unit, $Q_{g}^{(1, p, 1)}$ and $Q_{g}^{(1, p, 2)}$, respectively, denote the feedforward weight matrix of the encoder and decoder, and $b_{g}^{(1, p, 1)}$ and $b_{g}^{(1, p, 2)}$, respectively, denote the bias vector of the encoder and decoder. Noticeably, the DNN algorithm in [8] replaces the activation function with the unit operation to meet the additive property when multiple sources are distributed in different subregions. In contrast, the proposed method employs the phase difference as the input of the autoencoder and applies the ReLU function, which can approximate the nonlinear model and is suitable for the scenarios of multiple sources.

The dataset $\Psi_{\text {train }}^{(1, p)}=\left\{\left(u_{1}^{(1,0)}, v_{1}^{(1, p)}\right),\left(u_{2}^{(1,0)}, v_{2}^{(1, p)}\right), \cdots,(\right.$ $\left.\left.u_{G}^{(1,0)}, v_{G}^{(1, p)}\right)\right\}$ is applied to train the autoencoder of the $p$ th AOA subregion, where $v_{g}^{(1, p)}$ denotes the label (i.e., the expected output) of the $p$ th AOA subregion for the $g$ th training data. When the AOA of the data set $u_{g}^{(1,0)}$ belongs to the $p$ th subregion, the label set $v_{g}^{(1, p)}$ is $u_{g}^{(1,0)}$. Otherwise, the label 


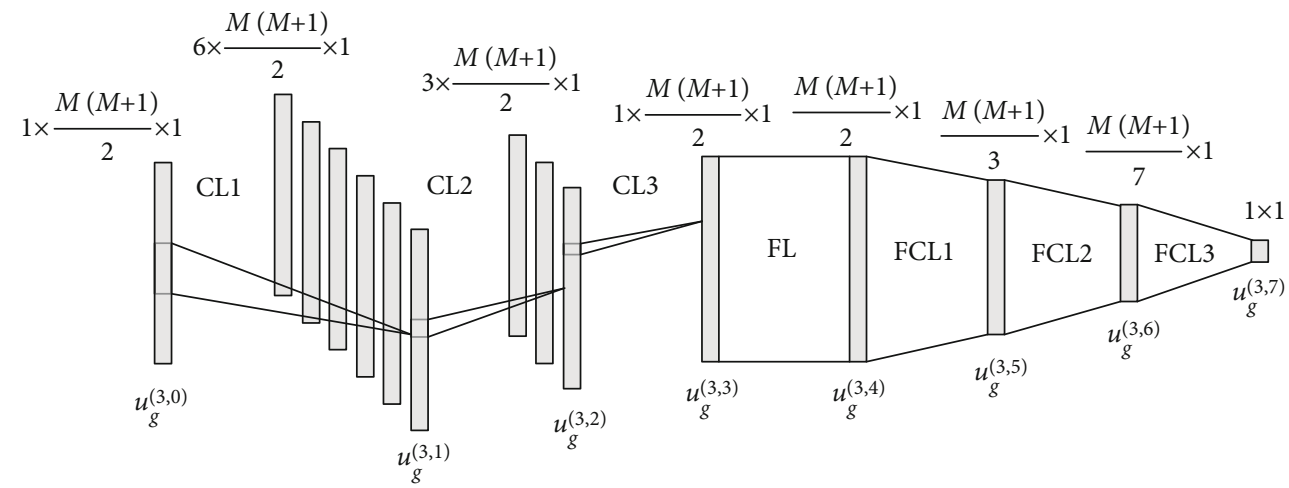

FIGURE 4: Regression CNN for the range estimation of the $g$ th data.

TABle 1: Main steps of the proposed method.

\begin{tabular}{|c|c|}
\hline & Algorithm for near-field source localization \\
\hline 1. & Construct the phase difference matrix of each near-field source in (8). \\
\hline 2. & $\begin{array}{c}\text { Extract the upper right counter-diagonal elements in (8) and perform the trained } p \text { th autoencoder to obtain the output of the } k \text { th } \\
\text { subregion } \widehat{u}_{k}^{(1, p, 2)} .\end{array}$ \\
\hline 3. & Perform the trained classification CNN to obtain the corresponding output of the $k$ th spatial spectrum $\widehat{u}_{k}^{(2, p, 5)}$. \\
\hline 4. & Splice the output of the $P$ AOA spatial spectrum and search the spatial peaks to determine the AOAs of the near-field sources. \\
\hline 5. & Construct a particular range vector in (17) to eliminate the estimated AOA of the $k$ th near-field source. \\
\hline 6. & Perform the trained regression CNN with $u_{k}^{(3,0)}$ to determine the corresponding range of the $k$ th near-field source. \\
\hline
\end{tabular}

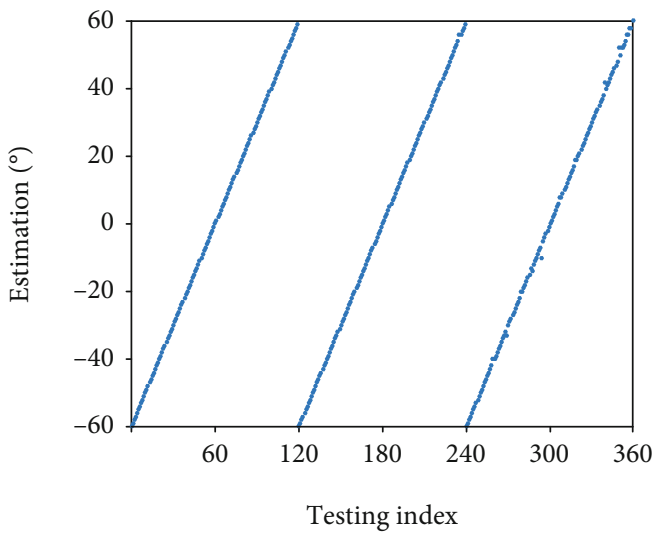

(a)

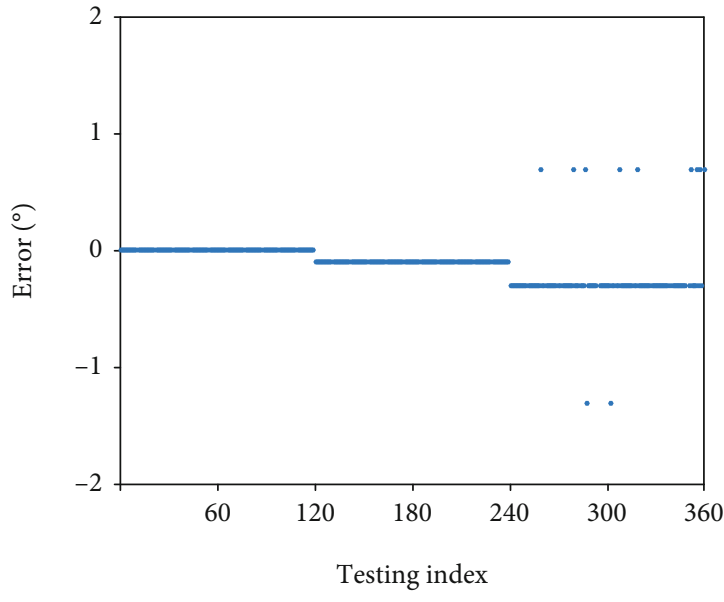

(b)

Figure 5: AOA of off-grid near-field sources. (a) Estimation. (b) Error.

set $v_{g}^{(1, p)}$ is an $M$-dimensional zero vector. Meanwhile, the mean square error (MSE) is used as the loss function and defined as

$$
\left\{\widehat{Q}_{g}^{(1, p, l)}, \widehat{b}_{g}^{(1, p, l)}\right\}_{l=1}^{2}=\underset{\left\{\boldsymbol{Q}_{g}^{(1, l, p)}, \boldsymbol{b}_{g}^{(1, l, p)}\right\}_{l=1}^{2}}{\arg \min } \frac{1}{2} \sum_{g=1}^{G}\left(u_{g}^{(1, p, 2)}-v_{g}^{(1, p)}\right)^{2} .
$$

\subsection{Classification CNN for AOA Estimation. After the auto-}

encoder is trained, the prediction for the $g$ th training data of the $p$ th autoencoder is

$$
\widehat{u}_{g}^{(1, p, 2)}=Q_{g}^{(1, p, 2)}\left(Q_{g}^{(1, p, 1)} u_{g}^{(1,0)}+b_{g}^{(1, p, 1)}\right)+b_{g}^{(1, p, 2)} .
$$

Based on the prediction of the autoencoder, we employ the classification $\mathrm{CNN}$ to obtain the spatial spectrum and estimate the AOAs of the near-field sources. Herein, the $\mathrm{CNN}$ of the $p$ th subregion for the $g$ th data is depicted in 


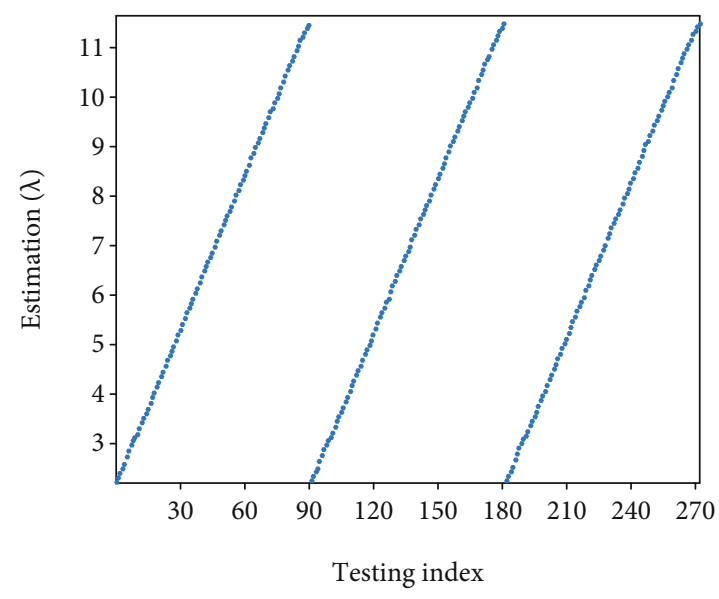

(a)

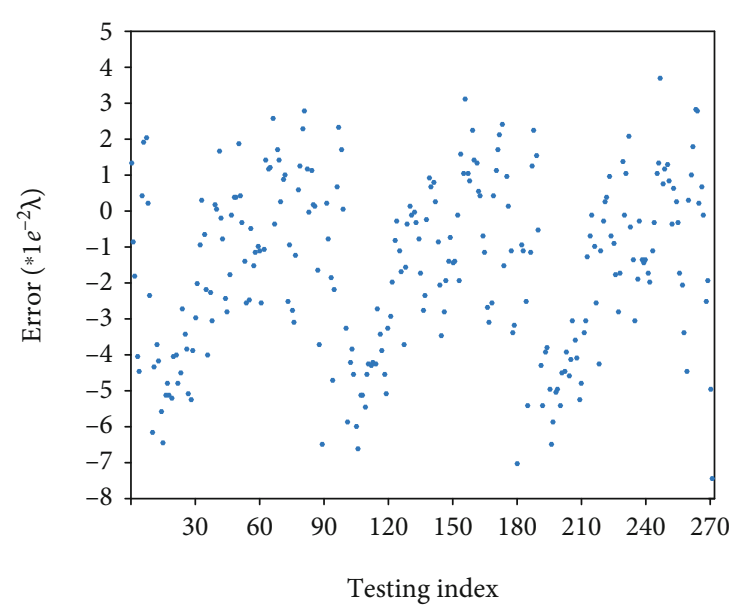

(b)

FIGURE 6: Range of off-grid near-field sources. (a) Estimation. (b) Error.

TABLE 2: Real location and estimated location of different sources.

\begin{tabular}{lcr}
\hline$K$ & $(\phi, \theta)$ & $(\widehat{\phi}, \widehat{\theta})$ \\
\hline 1 & $\left(30^{\circ}, 5 \lambda\right)$ & $\left(30^{\circ}, 5.02 \lambda\right)$ \\
3 & $\left(30^{\circ}, 5 \lambda\right)\left(-10^{\circ}, 8 \lambda\right)(-20,10 \lambda)$ & $\left(30^{\circ}, 5.02 \lambda\right)\left(-10^{\circ}, 7.97 \lambda\right)\left(-20^{\circ}, 10.00 \lambda\right)$ \\
5 & $\left(30^{\circ}, 5 \lambda\right)\left(-10^{\circ}, 8 \lambda\right)(-20,10 \lambda)\left(-25^{\circ}, 6 \lambda\right)\left(-50^{\circ}, 8 \lambda\right)$ & $\left(30^{\circ}, 5.02 \lambda\right)\left(-10^{\circ}, 7.97 \lambda\right)\left(-20^{\circ}, 10.00 \lambda\right)\left(-25^{\circ}, 6.02 \lambda\right)\left(-50^{\circ}, 8.00 \lambda\right)$ \\
\hline
\end{tabular}

Figure 3, which contains three convolution layers (CLs), one flatten layer (FL), and one fully connected layer (FCL).

Clearly, the output of the $l$ th CL is

$$
u_{g}^{(2, p, l)}=\Omega\left(\operatorname{Re} \operatorname{LU}\left(Q_{g}^{(2, p, l)} * u_{g}^{(2, p, l-1)}+b_{g}^{(2, p, l)}\right)\right) \quad l=1,2,3,
$$

where $u_{g}^{(2, p, 0)}=\widehat{u}_{g}^{(1, p, 2)}$, * denotes the convolution operation, $\Omega(\cdot)$ denotes the zero-padding operation to make the output size equal to the input size, and $Q_{g}^{(2, p, l)}$ and $b_{g}^{(2, p, l)}$, respectively, denote the convolution kernel and bias. Moreover, the $\zeta_{p}$-dimensional vector $u_{g}^{(2, p, 5)}$ denotes the output of the FCL and the $p$ th AOA subregion, which is given by

$$
u_{g}^{(2, p, 5)}=\operatorname{Tanh}\left(Q_{g}^{(2, p, 5)} u_{g}^{(2, p, 4)}+b_{g}^{(2, p, 5)}\right)
$$

where $\operatorname{Tanh}(\cdot)$ denotes the activation function of tangent hyperbolic and $Q_{g}^{(2, p, 5)}$ and $b_{g}^{(2, p, 5)}$, respectively, denote the feed-forward weight matrix and bias vector of FCL. Herein, MSE is also used as the loss function and the dataset $\Psi_{\text {train }}^{(2, p)}$ $=\left\{\left(\widehat{u}_{1}^{(1, p, 2)}, v_{1}^{(2, p)}\right),\left(\widehat{u}_{2}^{(1, p, 2)}, v_{2}^{(2, p)}\right), \cdots,\left(\widehat{u}_{G}^{(1, p, 2)}, v_{G}^{(2, p)}\right)\right\}$ is used to train the $\mathrm{CNN}$ for the $p$ th $\mathrm{AOA}$ subregion, where $v_{g}^{(2, p)}$ denotes the label (i.e., the corresponding spatial spectrum) of the $p$ th AOA subregion for the $g$ th training data.

Therefore, the AOA spatial spectrum of the near-field sources can be obtained by splicing the output of the $P$ AOA subregions, and the AOA of the near-field sources can be determined from the position of the spatial peaks.

3.4. Regression CNN for Range Estimation. Since the second part of the elements in (8) contains the range parameter of the near-field sources, and the first part of the counterdiagonal symmetric elements (e.g., $u_{m_{4}, m_{5}, g}$ and $u_{-m_{5}, m_{4}, g}$ ) are equal, we eliminate the AOA in (8) and apply the regression CNN to determine the range of the near-field sources. Herein, the CNN for the range estimation is depicted in Figure 4, which contains three CLs, one FL, and three FCLs.

To be specific, the output of the $l$ th CL is

$$
u_{g}^{(3, l)}=\Omega\left(\operatorname{Re} \operatorname{LU}\left(Q_{g}^{(3, l)} * u_{g}^{(3, l-1)}+b_{g}^{(3, l)}\right)\right) \quad l=1,2,3
$$

where the input of the 1 st CL is

$$
u_{g}^{(3,0)}=\frac{\left[u_{-M,-M+1, g}-u_{M-1, M, g}, u_{-M,-M+2, g}-u_{M-2, M, g}, \cdots, u_{m_{4}, m_{5}, g}-u_{-m_{5},-m_{4}, g}, \cdots, u_{-1,0, g}-u_{0,1, g}\right]^{T}}{\cos ^{2} \widehat{\theta}_{g}},
$$




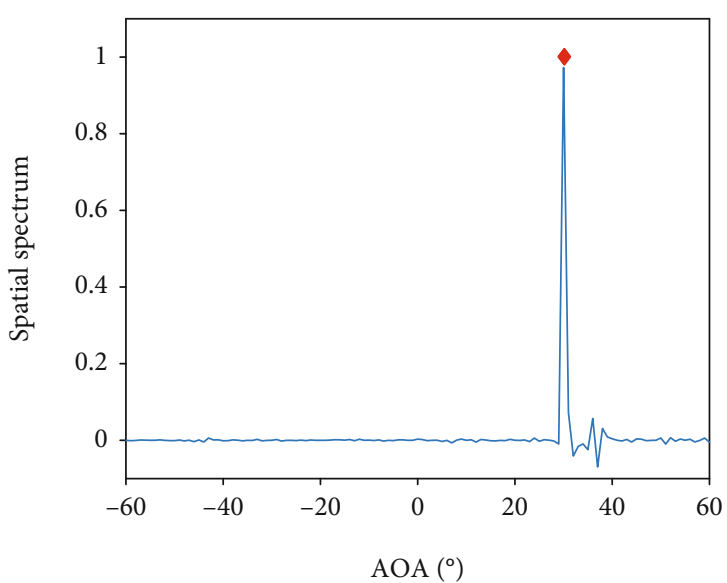

(a)

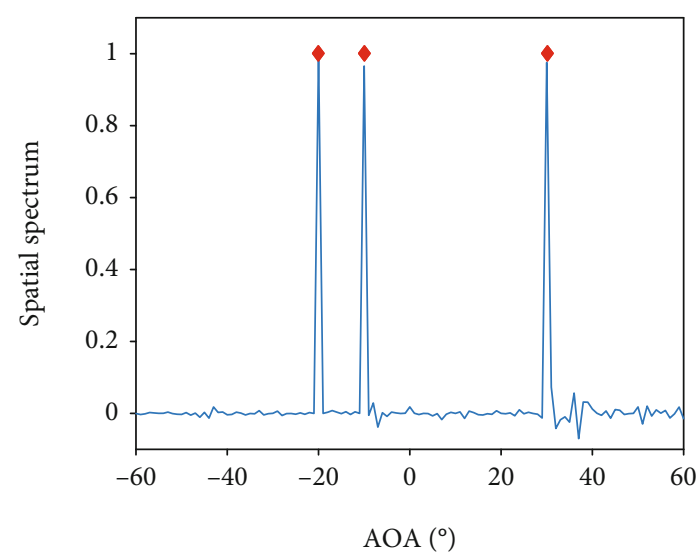

(b)

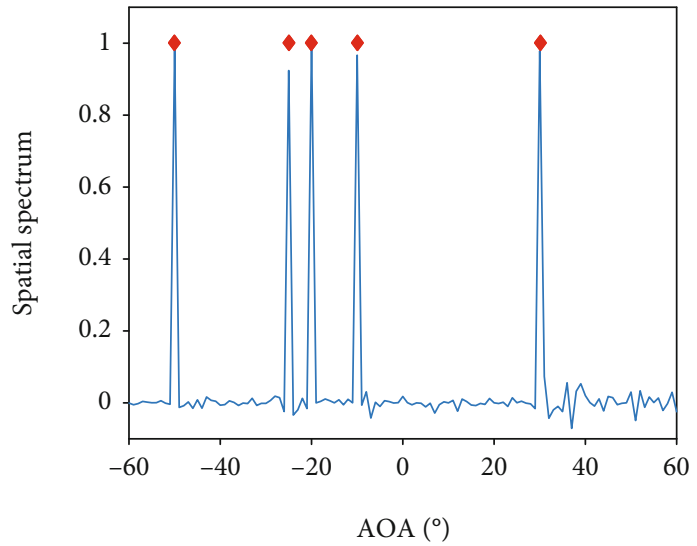

(c)

Figure 7: AOA spatial spectrum of different sources. (a) $K=1$. (b) $K=3$. (c) $K=5$.

for $m_{4}=-M,-M+1, \cdots,-1, m_{5}=m_{4}+1, m_{4}+2, \cdots, 0, \widehat{\theta}_{g}$ denotes the estimated AOA of the $g$ th data. Moreover, the output of the $l$ th FCL is

$$
u_{g}^{(3, l)}=\operatorname{ReLU}\left(Q_{g}^{(3, l)} u_{g}^{(3, l-1)}+b_{g}^{(3, l)}\right) \quad l=5,6,7
$$

Similarly, MSE is used as the loss function for each layer, and the dataset $\boldsymbol{\Psi}_{\text {train }}^{(3)}=\left\{\left(u_{1}^{(3,0)}, v_{1}^{(3)}\right),\left(u_{2}^{(3,0)}, v_{2}^{(3)}\right), \cdots,\left(u_{G}^{(3,0)}\right.\right.$, $\left.\left.v_{G}^{(3)}\right)\right\}$ is utilized to train the $\mathrm{CNN}$, where $v_{g}^{(3)}$ denotes the label (i.e., the corresponding range) for the $g$ th training data.

With respect to the computational complexity, we consider the major multiplications and additions in the networks. As for the autoencoder of each subregion, the computational complexity of encoder requires $M \times(M / 2)$ multiplications plus $M / 2$ additions, and that of decoder requires $(M / 2) \times M$ multiplications plus $M$ additions. As for the classification $\mathrm{CNN}$ for AOA estimation, the computational complexity of multiplications in CLs is $h_{1,1} \times 12 M$, $h_{1,2} \times 6 M$ and $h_{1,3} \times 3 M$, that of additions in CLs are $12 M$, $6 M$, and $3 M$, that of multiplication in FCL is $\zeta_{p} \times 3 M$, and that of addition in FCL is $\zeta_{p}$, where $h_{1,1}, h_{1,2}$, and $h_{1,3}$, respectively, denote the filter length of CLs in the classification
CNN. As for the regression CNN for range estimation, the computational complexity of multiplications in CLs is $h_{2,1}$ $\times 3 M(M+1), h_{2,2} \times 1.5 M(M+1)$, and $h_{2,3} \times 0.5 M(M+1)$, that of additions in CLs are $3 M(M+1), 1.5 M(M+1)$, and $0.5 M(M+1)$, that of multiplications in FCL are $(M(M+1)$ /3) $\times(M(M+1) / 2),(M(M+1) / 7) \times(M(M+1) / 3)$, and 1 $\times(M(M+1) / 7)$, and that of addition in FCL is $M(M+1) /$ 3, $M(M+1) / 7$, 1 , where $h_{2,1}, h_{2,2}$, and $h_{2,3}$, respectively, denote the filter length of CLs in the regression CNN.

Once the networks have been trained, the main steps of the proposed method to achieve near-field source localization are as shown in Table 1.

\section{Simulation Results}

In the simulations, the sensor number of SDNA is $13(M=6$ ), the AOA scope is from $60^{\circ}$ to $60^{\circ}$ which is divided into 12 subregions, and the near-field region is from $2.4 \lambda$ to $11.5 \lambda$. The AOA scope and near-field region are, respectively, uniformly sampled with intervals of $1^{\circ}$ and $0.1 \lambda$ to generate 11132 training groups, where the $\mathrm{AOAs}$ are set as $\left\{-60^{\circ}\right.$, $\left.-59^{\circ}, \ldots-1^{\circ}, 0^{\circ}, 1^{\circ}, \ldots, 60^{\circ}\right\}$ and the range parameters are set as $\{2.4 \lambda, 2.5 \lambda, \ldots, 11.5 \lambda\}$. Each group contains 10 training dataset with different SNR, where the snapshot number is 1024 and the SNR is randomly picked from $5 \mathrm{~dB}$ to $25 \mathrm{~dB}$. The 


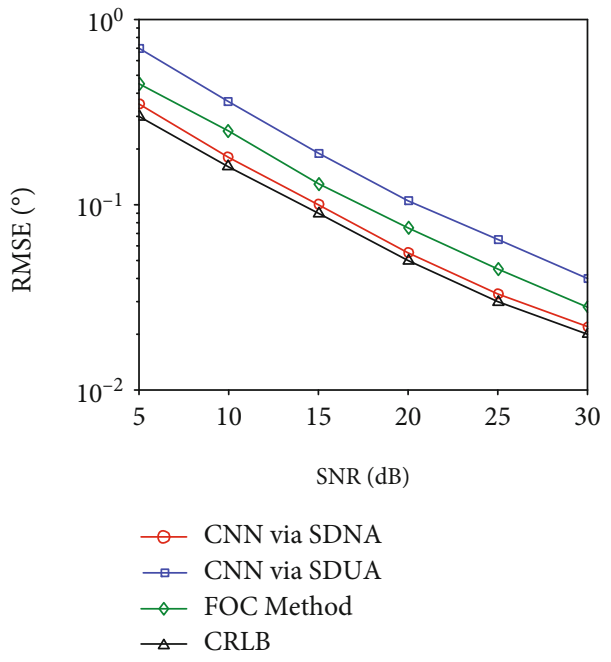

(a)

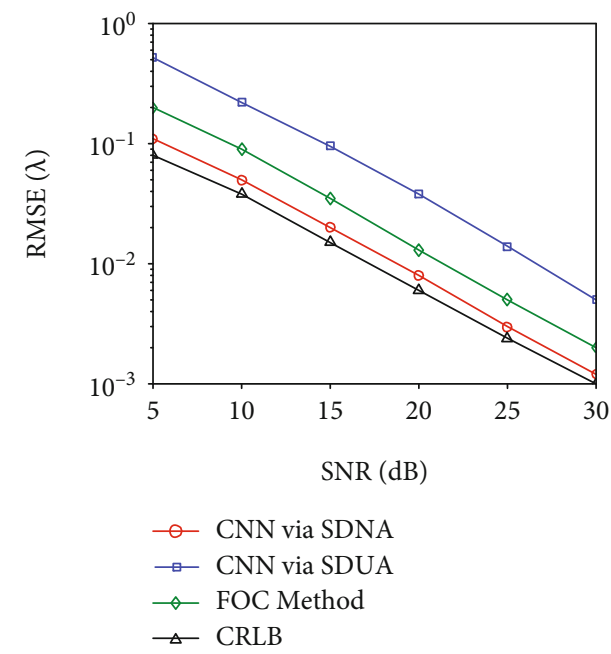

(b)

Figure 8: RMSEs versus SNR. (a) AOA. (b) Range.

networks are trained by Keras platform and updated with Adam optimizer, where the filter lengths of CLs in the classification $\mathrm{CNN}$ are 5, 4, and 3, while those in the regression $\mathrm{CNN}$ are 15,5 , and 3 , and the learning rate, minibatch size, and training epoch are set as $0.001,32$, and 300 , respectively.

4.1. Robustness of Parameter Estimation. In order to verify the robustness of AOA estimation, the AOA interval is set as $1^{\circ}$ to generate 3 testing groups with 360 off-grid nearfield sources, where the AOAs of the 1st group are $\left\{-59.99^{\circ}\right.$, $\left.-58.99^{\circ}, \ldots-0.99^{\circ}, 0.01^{\circ}, 1.01^{\circ}, \ldots, 59.01^{\circ}\right\}$, the 2 nd group are $\left\{-59.90^{\circ},-58.90^{\circ}, \ldots-0.90^{\circ}, 0.10^{\circ}, 1.10^{\circ}, \ldots,-59.10^{\circ}\right\}$, and the 3rd group are $\left\{-59.70^{\circ},-58.70^{\circ}, \ldots-0.70^{\circ}, 0.30^{\circ}, 1.30^{\circ}, \ldots\right.$, $\left.-59.30^{\circ}\right\}$; the ranges are all set to $5 \lambda$, and the SNR is randomly picked from $5 \mathrm{~dB}$ to $25 \mathrm{~dB}$. Figure 5 shows that the results are estimated to the adjacent grids if the AOA off-grid is $0.01^{\circ}$ or $0.1^{\circ}$, while $99.97 \%$ results are estimated to the adjacent grids if the AOA off-grid is $0.3^{\circ}$.

Similarly, the range interval is set as $0.1 \lambda$ to generate 3 testing groups with 273 off-grid near-field sources, where the ranges of the 1 st group are $\{2.41 \lambda, 2.51 \lambda, \ldots, 11.41 \lambda\}$, the 2 nd group are $\{2.43 \lambda, 2.53 \lambda, \ldots, 11.43 \lambda\}$, and the 3 rd group are $\{2.45 \lambda, 2.55 \lambda, \ldots, 11.45 \lambda\}$, and the AOAs are all set to $10^{\circ}$. Figure 6 displays that the estimation error is within $8 e^{-2} \lambda$, which proves that the proposed method is robust to parameter estimation.

4.2. Effectiveness for Different Number of Sources. Compared with the algorithm in [13] that utilizes the covariance matrix to train the networks in the case of the fixed number of sources, the proposed method employs the phase difference matrix of each source to train the networks. When the SNR is set as $10 \mathrm{~dB}$, Table 2 shows the real location and estimated location in the case of 1,3 , and 5 sources. Besides, Figure 7 displays the AOA spatial spectrum, where the diamondshaped points are the real AOAs and correspond to the spectrum peaks. Therefore, it can conclude that the proposed method is suitable for the localization scenarios with different number of sources.

4.3. RMSEs of Parameter Estimation. In order to further demonstrate the performance of near-field source localization, we compare the proposed method via the geometry of SDNA to that via symmetric double uniform array (SDUA) in the case of the same sensor number. Besides, we compare the proposed method to the state of the art fourth-order cumulant (FOC) method in [18] via the geometry of SDNA and the theoretical Cramér-Rao lower Bound (CRLB) via the geometry of SDNA.

The root mean square errors (RMSEs) of AOA and range parameters are given in Figure 8, where the locations of the near-field sources in the simulations are set as $\left(10^{\circ}, 4 \lambda\right)$ and $\left(20^{\circ}, 6.1 \lambda\right)$, and the RMSE at each SNR is determined by 500 independent Monte Carlo simulations. It can be seen that the RMSE of the proposed method via SDNA is lower than that via SDUA. Moreover, the proposed method outperforms the FOC method and can improve the accuracy of near-field source localization.

\section{Conclusions}

This letter exploits the geometry of SDNA to achieve the localization of near-field sources, where the autoencoders and classification CNNs are utilized to jointly determine the AOAs of near-field sources, and the regression $\mathrm{CNN}$ is employed to determine the range of near-field sources. Simulation results illustrate that the proposed method is robust to the parameter estimation of off-grid near-field sources. Besides, the proposed method applies the phase difference as the input of networks, which leads to the advantages for the localization scenarios with different number of nearfield sources. In addition, the proposed method via SNDA outperforms the FOC method and has higher accuracy than that via SNUA for near-field source localization. 


\section{Data Availability}

The raw/processed data required to reproduce these findings cannot be shared at this time as the data also forms part of an ongoing study.

\section{Conflicts of Interest}

The authors declare no conflict of interest.

\section{Authors' Contributions}

The main idea was proposed by Xiaolong Su and Zhen Liu; Panhe $\mathrm{Hu}$ and Zhenghui Gong conceived and designed the experiments; Xiaolong Su wrote the paper; Junpeng Shi and Xiang Li revised the paper.

\section{Acknowledgments}

This work was supported by the National Natural Science Foundation of China under Grant 62022091 and Grant 61921001.

\section{References}

[1] S. Chakrabarty and E. A. P. Habets, "Multi-speaker DOA estimation using deep convolutional networks trained with noise signals," IEEE Journal of Selected Topics in Signal Processing, vol. 13, no. 1, pp. 8-21, 2019.

[2] Y. Cao, T. Lv, Z. Lin, P. Huang, and F. Lin, "Complex ResNet aided DoA estimation for near-field MIMO systems," IEEE Transactions on Vehicular Technology, vol. 69, no. 10, pp. 11139-11151, 2020.

[3] D. Hu, Y. Zhang, L. He, and J. Wu, "Low-complexity deeplearning-based DOA estimation for hybrid massive MIMO systems with uniform circular arrays," IEEE Wireless Communications Letters, vol. 9, no. 1, pp. 83-86, 2020.

[4] H. Chen, W. Wang, and W. Liu, "Joint DOA, range, and polarization estimation for rectilinear sources with a COLD array," IEEE Wireless Communications Letters, vol. 8, no. 5, pp. 13981401, 2019.

[5] H. Chen, W. Zhu, W. Liu et al., "RARE-based localization for mixed near-field and far-field rectilinear sources," Digital Signal Processing, vol. 85, pp. 54-61, 2019.

[6] G. Liu and X. Sun, "Spatial differencing method for mixed farfield and near-field sources localization," IEEE Signal Processing Letters, vol. 21, pp. 1331-1335, 2014.

[7] W. Zuo, J. Xin, N. Zheng, and A. Sano, "Subspace-based localization of far-field and near-field signals without eigendecomposition," IEEE Transactions on Signal Processing, vol. 66, no. 17, pp. 4461-4476, 2018.

[8] Z. Liu, C. Zhang, and P. S. Yu, "Direction-of-arrival estimation based on deep neural networks with robustness to array imperfections," IEEE Transactions on Antennas and Propagation, vol. 66, no. 12, pp. 7315-7327, 2018.

[9] L. Wu, Z. Liu, and Z. Huang, "Deep convolution network for direction of arrival estimation with sparse prior," IEEE Signal Processing Letters, vol. 26, no. 11, pp. 1688-1692, 2019.

[10] W. Zuo, J. Xin, H. Ohmori, N. Zheng, and A. Sano, "Subspacebased algorithms for localization and tracking of multiple near-field sources," IEEE Journal of Selected Topics in Signal Processing, vol. 13, no. 1, pp. 156-171, 2019.

[11] X. Su, Z. Liu, T. Liu, B. Peng, X. Chen, and X. Li, “An SOSbased algorithm for localization of multiple near-field sources using uniform circular array," IEEE Sensors Letters, vol. 3, no. 11, pp. 1-4, 2019.

[12] C. Guanghui, Z. Xiaoping, J. Shuang, Y. Anning, and L. Qi, "High accuracy near-field localization algorithm at low SNR using fourth-order cumulant," IEEE Communications Letters, vol. 24, no. 3, pp. 553-557, 2020.

[13] W. Liu, J. Xin, W. Zuo, J. Li, N. Zheng, and A. Sano, "Deep learning based localization of near-field sources with exact spherical wavefront model," in 2019 27th European Signal Processing Conference (EUSIPCO), pp. 1-5, A Coruna, Spain, 2019.

[14] P. Pal and P. P. Vaidyanathan, "Nested arrays: a novel approach to array processing with enhanced degrees of freedom," IEEE Transactions on Signal Processing, vol. 58, no. 8, pp. 4167-4181, 2010.

[15] K. Han and A. Nehorai, "Nested array processing for distributed sources," IEEE Signal Processing Letters, vol. 21, pp. 1111-1114, 2014.

[16] P. Shi, F. Wen, and T. Liu, "Nested MIMO radar: coarrays, tensor modeling and angle estimation," IEEE Transactions on Aerospace and Electronic Systems, vol. 57, pp. 573-585, 2021.

[17] Z. Zheng, M. Fu, W. Wang, S. Zhang, and Y. Liao, "Localization of mixed near-field and far-field sources using symmetric double-nested arrays," IEEE Transactions on Antennas and Propagation, vol. 67, no. 11, pp. 7059-7070, 2019.

[18] T. Shu, L. Li, and J. He, "Near-field source localization with two-level nested arrays," IEEE Communications Letters, vol. 24, no. 11, pp. 2488-2492, 2020. 